\section{Full-wave equivalent circuit for the analysis of multilayered microwave structures with anisotropic layers}

\section{Kamra ${ }^{\bowtie}$ and A. Dreher}

A simple formalism is presented as a basis to analyse planar microwave structures with uniaxial anisotropic materials having either electric or magnetic anisotropy or both. It involves a generalised relation of the field components of the multilayered structure, which can be represented by a full-wave hybrid matrix for each layer. Thus, for stratified structures, the system equation can be obtained by using a full-wave equivalent circuit. The application is demonstrated by analysing the well-known example of a two-layer waveguide filled with different uniaxial anisotropic dielectric material.

Introduction: Anisotropic materials are widely used in the field of microwave engineering and integrated optics. Because of the technological advancements, it is becoming more and more easy to produce anisotropic films and substrates. Hence, there is also a need to better characterise the anisotropic medium and to develop efficient techniques to model the components.

Several numerical techniques and commercial software tools are available to analyse planar microwave structures such as waveguides, microstrip lines and antennas [1]. Also, significant work has been done by several authors using different approaches for considering anisotropy in the material [2-4]. In this Letter, the focus is on multilayered planar structures having numerous interfaces and arbitrarily thin layers as given in [5] for the isotropic case. The most complicated task while analysing is to set up the system matrix. It is possible to derive the system equation or dyadic Green's function by using a full-wave equivalent circuit (FWEC), where each dielectric layer is represented by a hybrid matrix, which relates all the tangential field components at the interfaces (Fig. 1). The current sources represent metallisations between the layers, and shorts or admittances are used for closed or open top and bottom layers. This equivalent circuit was already applied successfully for the analytical and numerical analysis of waveguides and microstrip antennas in different coordinate systems $[6,7]$.

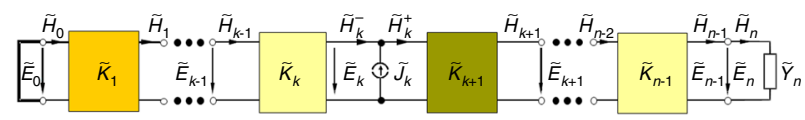

Fig. 1 Full-wave equivalent circuit of a general planar microwave structure with stratified dielectric

This Letter now describes the derivation of the hybrid-matrix elements for uniaxial anisotropic structures. The application of the extended equivalent circuit is demonstrated by analysing a simple stratified microwave structure like a waveguide. The results are compared with those obtained with commercial software.

Analysis: To characterise the anisotropic_medium, the analysis starts from permittivity $(\overline{\bar{\varepsilon}})$ and permeability $(\overline{\bar{\mu}})$ tensor with optic axis in the $z$-direction

$$
\overline{\bar{\varepsilon}}=\left[\begin{array}{ccc}
\varepsilon_{x} & 0 & 0 \\
0 & \varepsilon_{x} & 0 \\
0 & 0 & \varepsilon_{z}
\end{array}\right], \quad \overline{\bar{\mu}}=\left[\begin{array}{ccc}
\mu_{x} & 0 & 0 \\
0 & \mu_{x} & 0 \\
0 & 0 & \mu_{z}
\end{array}\right]
$$

Maxwell's equations in the space-frequency domain and for a sourcefree and homogeneous medium are given in their differential form by

$$
\begin{gathered}
\nabla \times \boldsymbol{E}(x, y, z)=-\mathrm{j} \omega \mu_{0} \overline{\bar{\mu}} \cdot \boldsymbol{H}(x, y, z), \\
\nabla \times \boldsymbol{H}(x, y, z)=\mathrm{j} \omega \varepsilon_{0} \overline{\bar{\varepsilon}} \cdot \boldsymbol{E}(x, y, z),
\end{gathered}
$$

where $\boldsymbol{E}$ and $\boldsymbol{H}$ are electric and magnetic field vectors, $\omega$ is the angular frequency, $\mu_{0}$ is the free-space permeability and $\varepsilon_{0}$ is the free-space permittivity. To simplify the analysis, space variables are normalised by the free space wave number $k_{0}$, and $\eta_{0} H$ is replaced by $H$, where $\eta_{0}=\sqrt{\mu_{0} / \varepsilon_{0}}$, is the intrinsic impedance of the free space. So in the extended form, Faraday's law (2a) and Ampere's law (2b) give the field relations as

$$
\begin{aligned}
\frac{\partial}{\partial y} E_{z}-\frac{\partial}{\partial z} E_{y} & =-\mathrm{j} \mu_{x} H_{x}, & \frac{\partial}{\partial y} H_{z}-\frac{\partial}{\partial z} H_{y} & =\mathrm{j} \varepsilon_{x} E_{x}, \\
\frac{\partial}{\partial z} E_{x}-\frac{\partial}{\partial x} E_{z} & =-\mathrm{j} \mu_{x} H_{y}, & \frac{\partial}{\partial z} H_{x}-\frac{\partial}{\partial x} H_{z} & =\mathrm{j} \varepsilon_{x} E_{y}, \\
\frac{\partial}{\partial x} E_{y}-\frac{\partial}{\partial y} E_{x} & =-\mathrm{j} \mu_{z} H_{z}, & \frac{\partial}{\partial x} H_{y}-\frac{\partial}{\partial y} H_{x} & =\mathrm{j} \varepsilon_{z} E_{z} .
\end{aligned}
$$

As the optical axis is in the $z$-direction, we can take $E_{z}$ and $H_{z}$ as two independent field components. Therefore, on rearranging (3), the other field components can be calculated using the relation

$$
\left(\frac{\partial^{2}}{\partial z^{2}}+\varepsilon_{x} \mu_{x}\right)\left[\begin{array}{c}
E_{x} \\
H_{x} \\
E_{y} \\
H_{y}
\end{array}\right]=\left[\begin{array}{cc}
\frac{\partial}{\partial x} \frac{\partial}{\partial z} & -\mathrm{j} \mu_{x} \frac{\partial}{\partial y} \\
\mathrm{j} \varepsilon_{x} \frac{\partial}{\partial y} & \frac{\partial}{\partial x} \frac{\partial}{\partial z} \\
\frac{\partial}{\partial y} \frac{\partial}{\partial z} & \mathrm{j} \mu_{x} \frac{\partial}{\partial x} \\
-\mathrm{j} \varepsilon_{x} \frac{\partial}{\partial x} & \frac{\partial}{\partial y} \frac{\partial}{\partial z}
\end{array}\right]\left[\begin{array}{c}
E_{z} \\
H_{z}
\end{array}\right] .
$$

Similarly, for the case of the optic axis in the $x$ - or $y$-direction, the independent field components will be $E_{x}, H_{x}$ and $E_{y}, H_{y}$, respectively. The whole analysis procedure will be same as shown here for the case of the $z$ optical axis.

Now consider a stratified dielectric in the $z$-direction. The source-free wave equation can be written as

$$
\left(\frac{\partial^{2}}{\partial x^{2}}+\frac{\partial^{2}}{\partial y^{2}}+\frac{\partial^{2}}{\partial z^{2}}+K^{2}\right) \psi=0
$$

where $\psi$ represents two independent field components, i.e. $E_{z}, H_{z}$. Transforming (5) into spectral domain leads to the ordinary differential equation

$$
\left(\frac{\partial^{2}}{\partial z^{2}}-k_{z}^{2}\right) \tilde{\psi}=0
$$

with $k_{z}^{2}=k_{x}^{2}-\left(k^{2}-k_{y}^{2}\right)$. In contrast to isotropic media, it must be taken into account, that in the uniaxial anisotropic case the propagation constant $k$ has two roots. One represents TE waves (ordinary waves) and the other represents TM waves (extraordinary waves). Therefore, the dispersion relation for the $E_{z}$ (TM mode) and $H_{\mathrm{z}}$ (TE mode) is obtained as

$$
k_{z e}=\sqrt{\frac{\varepsilon_{x}}{\varepsilon_{z}}\left(-\mu_{x} \varepsilon_{z}+k_{s}^{2}\right)} \text { and } k_{z h}=\sqrt{\frac{\mu_{x}}{\mu_{z}}\left(-\mu_{z} \varepsilon_{x}+k_{s}^{2}\right)}
$$

respectively with $k_{s}=\sqrt{k_{x}^{2}+k_{y}^{2}}$. Then the solution of (6) within an arbitrary layer $k$ can be written in the form

$$
\tilde{E}_{z k}=A e^{k_{z e} z}+B e^{-k_{z e} z} \text { and } \tilde{H}_{z}=C e^{k_{z h} z}+D e^{-k_{z h} z}
$$

Depending on the roots for $E_{z}$ and $H_{z}$, the transverse field components can be written in spectral domain as

$$
\begin{gathered}
\tilde{E}_{x}=-\frac{\mathrm{j} k_{x} \varepsilon_{z}}{\varepsilon_{x} k_{s}^{2}} \frac{\partial}{\partial z} \tilde{E}_{z}-\frac{k_{y} \mu_{z}}{k_{s}^{2}} \tilde{H}_{z}, \\
\tilde{E}_{y}=-\frac{\mathrm{j} k_{y} \varepsilon_{z}}{\varepsilon_{x} k_{s}^{2}} \frac{\partial}{\partial z} \tilde{E}_{z}+\frac{k_{x} \mu_{z}}{k_{s}^{2}} \tilde{H}_{z}, \\
\tilde{H}_{x}=\frac{k_{y} \varepsilon_{z}}{k_{s}^{2}} \tilde{E}_{z}-\frac{\mathrm{j} k_{x} \mu_{z}}{\mu_{x} k_{s}^{2}} \frac{\partial}{\partial z} \tilde{H}_{z}, \\
\tilde{H}_{y}=-\frac{k_{x} \varepsilon_{z}}{k_{s}^{2}} \tilde{E}_{z}-\frac{\mathrm{j} k_{y} \mu_{z}}{\mu_{x} k_{s}^{2}} \frac{\partial}{\partial z} \tilde{H}_{z},
\end{gathered}
$$


On using (8) and (9), the transverse field components can be rewritten as

$$
\left[\begin{array}{c}
\tilde{E}_{x} \\
\tilde{H}_{x} \\
\tilde{E}_{y} \\
\tilde{H}_{y}
\end{array}\right]=\left[\begin{array}{cccc}
\tilde{Q}_{x}^{A} & \tilde{Q}_{x}^{B} & \tilde{Q}_{x}^{C} & \tilde{Q}_{x}^{D} \\
\tilde{G}_{x}^{A} & \tilde{G}_{x}^{B} & \tilde{G}_{x}^{C} & \tilde{G}_{x}^{D} \\
\tilde{Q}_{y}^{A} & \tilde{Q}_{y}^{B} & \tilde{Q}_{y}^{C} & \tilde{Q}_{y}^{D} \\
\tilde{G}_{y}^{A} & \tilde{G}_{y}^{B} & \tilde{G}_{y}^{C} & \tilde{G}_{y}^{D}
\end{array}\right]\left[\begin{array}{c}
A \\
B \\
C \\
D
\end{array}\right] .
$$

Now to obtain the relation between the fields at the interfaces $k-1$ and $k$ bounding the layer $k$, we write

$$
\widetilde{\boldsymbol{E}}_{k}=\left[\begin{array}{c}
\tilde{E}_{x} \\
\tilde{E}_{y}
\end{array}\right], \quad \widetilde{\boldsymbol{H}}_{k}=\left[\begin{array}{c}
\tilde{H}_{y} \\
\tilde{H}_{x}
\end{array}\right], \quad \widetilde{\boldsymbol{F}}=\left[\begin{array}{c}
A_{k} \\
B_{k} \\
C_{k} \\
D_{k}
\end{array}\right],
$$

and

$$
\left[\begin{array}{c}
\widetilde{\boldsymbol{E}}_{k-1} \\
\widetilde{\boldsymbol{H}}_{k-1}
\end{array}\right]=\tilde{M}_{k-1} \cdot \boldsymbol{F}, \quad\left[\begin{array}{c}
\widetilde{\boldsymbol{E}}_{k} \\
\widetilde{\boldsymbol{H}}_{k}
\end{array}\right]=\tilde{M}_{k} \cdot \boldsymbol{F},
$$

with

$$
\begin{aligned}
& \tilde{Q}_{x}^{A}=-\mathrm{j} \varepsilon_{z} k_{x} k_{z e} e^{k_{z z} z} /\left(\varepsilon_{x} k_{s}^{2}\right), \quad \tilde{Q}_{x}^{B}=\mathrm{j} \varepsilon_{z} k_{x} k_{z e} e^{-k_{z z} z} /\left(\varepsilon_{x} k_{s}^{2}\right), \\
& \tilde{Q}_{x}^{C}=-\mu_{z} k_{y} e^{k_{z h} z} /\left(k_{s}^{2}\right), \quad \tilde{Q}_{x}^{D}=-\mu_{z} k_{y} e^{-k_{z h} z} /\left(k_{s}^{2}\right), \\
& \tilde{Q}_{y}^{A}=-\mathrm{j} \varepsilon_{z} k_{y} k_{z e} e^{k_{z e} z} /\left(\varepsilon_{x} k_{s}^{2}\right), \quad \tilde{Q}_{y}^{B}=j \varepsilon_{z} k_{y} k_{z e} e^{-k_{z z} z} /\left(\varepsilon_{x} k_{s}^{2}\right), \\
& \tilde{Q}_{y}^{C}=\mu_{z} k_{x} e^{k_{z z} z} /\left(k_{s}^{2}\right), \quad \tilde{Q}_{y}^{D}=\mu_{z} k_{x} e^{-k_{z h} z} /\left(k_{s}^{2}\right), \\
& \tilde{G}_{x}^{A}=\varepsilon_{z} k_{y} e^{k_{z z} z} /\left(k_{s}^{2}\right), \quad \tilde{G}_{x}^{B}=\varepsilon_{z} k_{y} e^{-k_{z e} z} /\left(k_{s}^{2}\right), \\
& \tilde{G}_{x}^{C}=-\mathrm{j} \mu_{z} k_{x} k_{z h} e^{k_{z h} z} /\left(\mu_{x} k_{s}^{2}\right), \quad \tilde{G}_{x}^{D}=\mathrm{j} \mu_{z} k_{x} k_{z h} e^{-k_{z h} z} /\left(\mu_{x} k_{s}^{2}\right), \\
& \tilde{G}_{y}^{A}=-\varepsilon_{z} k_{x} e^{k_{z z} z} /\left(k_{s}^{2}\right), \quad \tilde{G}_{y}^{B}=-\varepsilon_{z} k_{x} e^{-k_{z e} z} /\left(k_{s}^{2}\right), \\
& \tilde{G}_{y}^{C}=-\mathrm{j} \mu_{z} k_{y} k_{z h} e^{k_{z h} z} /\left(\mu_{x} k_{s}^{2}\right), \quad \tilde{G}_{y}^{D}=\mathrm{j} \mu_{z} k_{y} k_{z h} e^{-k_{z h} z} /\left(\mu_{x} k_{s}^{2}\right) .
\end{aligned}
$$

On eliminating the unknown vector $\boldsymbol{F}$ from (12), the relation for the field components at the interfaces of the layer $k$ with normalised thickness $d_{k}$ can be written as

$$
\left[\begin{array}{c}
\widetilde{\boldsymbol{E}}_{k-1} \\
\widetilde{\boldsymbol{H}}_{k-1}
\end{array}\right]=\widetilde{\boldsymbol{K}}_{k}\left[\begin{array}{c}
\widetilde{\boldsymbol{E}}_{k} \\
\widetilde{\boldsymbol{H}}_{k}
\end{array}\right],
$$

with the hybrid matrix

$$
\widetilde{\boldsymbol{K}}_{k}=\tilde{M}_{k-1} \cdot \tilde{M}_{k}^{-1}=\left[\begin{array}{cc}
\tilde{\boldsymbol{V}}_{k} & \widetilde{\boldsymbol{Z}}_{k} \\
\tilde{\boldsymbol{Y}}_{k} & \widetilde{\boldsymbol{B}}_{k}
\end{array}\right] .
$$

Multiple layers can be taken into account by simple matrix multiplication.

After applying the theory of FWEC, the dyadic Green's function can be found in the spectral domain. The system equation can be written in the form

$$
\widetilde{\boldsymbol{G}} \cdot \widetilde{\boldsymbol{J}}=\widetilde{\boldsymbol{E}}
$$

where $\widetilde{\boldsymbol{G}}$ represents the dyadic Green's function and $\widetilde{\boldsymbol{J}}$ and $\widetilde{\boldsymbol{E}}$ represent electric current density and electric field in the interfaces, respectively.

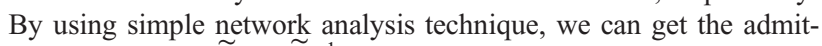
tance of the system $\widetilde{\boldsymbol{Y}}$ (or $\widetilde{\boldsymbol{G}}^{-1}$ ) [5].

Application: To validate this approach, a two-layer waveguide filled with anisotropic dielectric material stratified in the $z$-direction is analysed. The basic geometry (with $a=12.7 \mathrm{~mm}, d_{1}=0.1 a, d_{2}=0.9 a$, $\left.\varepsilon_{r 1}=(9.4,9.4,11.6), \varepsilon_{r 2}=1\right)$ and the propagation constant $k_{y}$ for the five lowest-order modes of the waveguide as a function of frequency are shown in Fig. 2. Fig. 3 gives the corresponding equivalent circuit from which the system equation is simply obtained as [5]

$$
\widetilde{\boldsymbol{Y}} \cdot \widetilde{\boldsymbol{E}}=0 \text {, where } \widetilde{\boldsymbol{Y}}=\widetilde{\boldsymbol{Z}}_{1}^{-1} \widetilde{\boldsymbol{V}}_{1}+\widetilde{\boldsymbol{B}}_{2} \widetilde{\boldsymbol{Z}}_{2}^{-1} .
$$

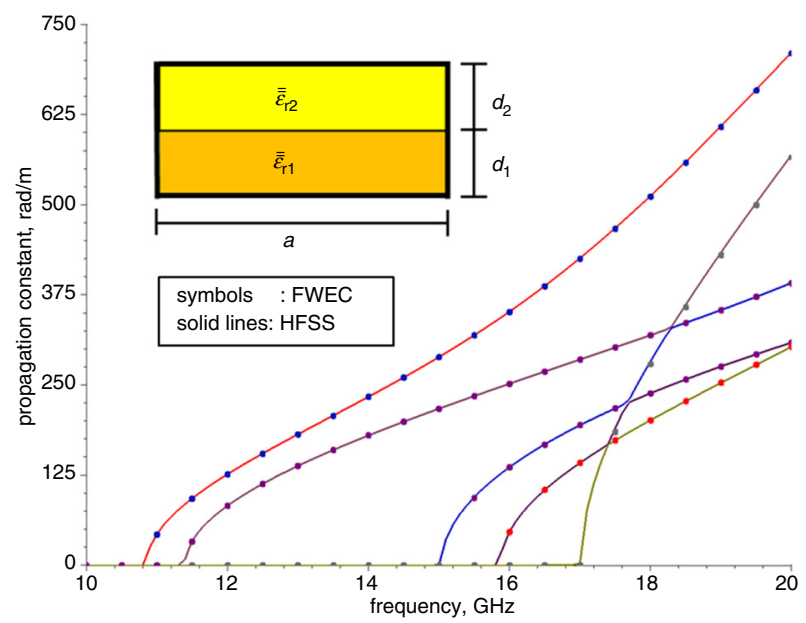

Fig. 2 Dispersion curve for the five lowest order modes of a two-layer waveguide (enclosed by ideal conductors) filled with anisotropic material

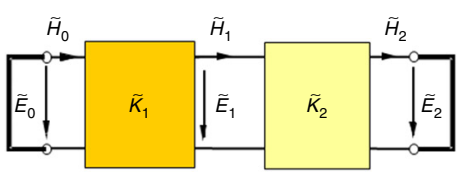

Fig. 3 Equivalent circuit for a two-layer waveguide

The propagation constant can be found by solving the indirect eigenvalue problem, i.e. $\operatorname{det}(\widetilde{\boldsymbol{Y}})=0$. Fig. 2 also depicts the comparison between the results obtained from our code using FWEC and the commercial software Ansys HFSS. A very good agreement is obtained; the difference between both results is found to be $\simeq 0.2 \%$.

Conclusion: Based on the Fourier transformation of electromagnetic field components, a new formulation of the tangential field relations on the interfaces of stratified anisotropic planar microwave structures in has been presented. Other microwave structures such as striplines and microstrip antennas can also be analysed using this FWEC with suitable numerical methods, where the dyadic Green's function is required. This formalism reduces the procedure to set up the system equation to simple circuit analysis and matrix multiplications.

(C) The Institution of Engineering and Technology 2017

Submitted: 25 September 2017

doi: 10.1049/el.2017.3668

One or more of the Figures in this Letter are available in colour online.

V. Kamra and A. Dreher (Institute of Communications and Navigation, German Aerospace Center (DLR), Oberpfaffenhofen, 82234 Wessling, Germany)

凶 E-mail: Veenu.Kamra@dlr.de

\section{References}

1 Itoh, T.: 'Numerical techniques for microwave and millimeter-wave passive structures' (John Wiley \& Sons, New York, 1989)

2 Pregla, R.: 'Analysis of electromagnetic fields and waves: the method of lines' (John Wiley \& Sons, Chichester, UK, 2008)

3 Krowne, C.: 'Determination of the green's function in the spectral domain using a matrix method: application to radiators or resonators immersed in a complex anisotropic layered medium', Trans. Antennas Propag., 1986, 34, (2), pp. 247-253

4 Mesa, F., Marqués, R., and Horno, M.: 'On the computation of the complete spectral green's dyadic for layered bianisotropic structure', Trans. Microw. Theory Tech., 1998, 46, (8), pp. 1158-1164

5 Dreher, A.: 'A new approach to dyadic green's function in spectral domain', Trans. Antennas Propag., 1995, 43, (11), pp. 1297-1302

6 Dreher, A., and Pregla, R.: 'Full-wave analysis of radiating planar resonators with the method of lines', Trans. Microw. Theory Tech., 1993, 41, (8), pp. 1363-1368

7 Thiel, M., and Dreher, A.: 'Dyadic green's function of multilayer cylindrical closed and sector structures for waveguide, microstrip-antenna and network analysis', Trans. Microw. Theory Tech., 2002, 50, (11), pp. 2576-2579 